\title{
El ocio en el medio natural como promotor de la conexión emocional con la naturaleza. Un estudio en clave ambiental con adolescentes pontevedreses (Galicia-España)
}

\author{
Leisure Time in Natural Environment as a \\ Promoter of Emotional Connection with Nature. \\ An Environmental Study with Teenagers in \\ Pontevedra (Galicia-Spain)
}

\section{Rubén Martínez García, María Belén Caballo Villar, Laura Varela Crespo}

Universidad de Santiago de Compostela

\section{Resumen}

\begin{abstract}
Los jóvenes cada vez tienen menos ocio en la naturaleza. Esta realidad se vincula con el despertar de la conciencia ambiental, pues el ocio en estos entornos promueve una mayor conexión emocional con la naturaleza, lo que influye en las actitudes y comportamientos proambientales. El objetivo del presente trabajo fue analizar la influencia que ejerce la experiencia de ocio en espacios naturales en el grado de conexión emocional con la naturaleza en una muestra de alumnos de educación secundaria de la provincia de Pontevedra (Galicia, España). Asimismo, se estudió el rol de variables como el lugar de residencia y el género. A partir de un muestreo por cuotas cruzadas se aplicó un cuestionario elaborado ad hoc con 683 casos válidos. Los datos informan que los adolescentes que tienen más experiencias de ocio en entornos naturales presentan un mayor grado de conectividad; el alumnado del ámbito rural muestra valores más elevados; y que son las estudiantes las que presentan una conectividad más alta. En conclusión, se subraya la necesidad de potenciar en los más jóvenes el ocio en entornos naturales como estrategia para restituir la armonía entre el ser humano y la naturaleza.
\end{abstract}

Palabras clave: adolescencia, conexión con la naturaleza, educación ambiental, ocio.

Correspondencia a:

Rubén Martínez García

Rúa Prof. Vicente Fráiz Andón, s/n. CP: 15782, Santiago de Compostela, España ruben.martinez.garcia@usc.es

Financiamiento asociado: Ayudas para la Formación del Profesorado Universitario (FPU) financiadas por el Ministerio de Ciencia, Innovación y Universidades del Gobierno de España.

Este trabajo emana de la tesis doctoral "Educación, ocio y espacios naturales. Un estudio con el alumnado de educación secundaria de la provincia de Pontevedra" defendida en la Universidad de Santiago de Compostela en el año 2019.

(c) 2020 PEL, http://www.pensamientoeducativo.org - http://www.pel.cl 


\section{Abstract}

Young people spend progressively less leisure time in contact with nature. This situation is closely related to the awakening of environmental awareness, since leisure time in natural environments fosters a greater emotional connection with nature, which influences proenvironmental attitudes and behaviors. The objective of this research is to analyze the influence of leisure experiences in natural spaces on the degree of emotional connection with nature among a group of secondary education students in Pontevedra (Galicia, Spain). The role of variables such as place of residence and gender in this emotional connection will also be analyzed. An ad hoc questionnaire was applied based on cross-sectional quota sampling, obtaining a total of 683 valid cases. The data obtained shows that those teenagers who have more leisure experiences in natural environments have a higher degree of emotional connection with nature. Likewise, the place of residence influences the degree of connection with nature, with the highest values being for students from rural areas. In addition, female students have significantly higher connectivity. In conclusion, it is necessary to enhance these leisure experiences in nature among the youngest students as a strategy to restore harmony between human beings and nature.

Keywords: connection with nature, environmental education, leisure, teenagers.

\section{Introducción}

La literatura científica publicada en los últimos años deja constancia de que el ocio de la infancia y la adolescencia está menos vinculado con la naturaleza que el de las generaciones anteriores (Clements, 2004; Henderson \& Bialeschki, 2008; Karsten, 2005; Louv, 2018; Soga, Gaston, Yamaura, Kurisu \& Hanaki, 2016). Lejos de ser una simple percepción social, son diversas las investigaciones que han puesto de manifiesto esta realidad. A modo de ejemplo, en EE.UU., en el periodo transcurrido entre 1993 y 1997 se produjo en los menores de 9 a 12 años una disminución del 50\% en actividades de ocio al aire libre como paseos, pesca, juegos en la playa, etc.; paralelamente su tiempo libre disminuyó más de siete horas semanales entre 1981 y 1997 (Hofferth \& Sandberg, 2001). Asimismo, en los años transcurridos entre 1997 y 2003 ese tiempo libre se redujo dos horas más (Hofferth \& Curtin, 2006), lo que implica un total de nueve horas menos de juego libre en un lapso de veinte años. De forma análoga, en Espańa y en el resto de países europeos, desde los años 70 del siglo pasado el tiempo que disfruta la infancia jugando de forma autónoma ha disminuido un $90 \%$ y el tiempo libre se ha visto reducido en torno a 15 horas semanales. A ello hay que añadir que solo el $29 \%$ de los niños y niñas de hoy experimentan momentos de juego al aire libre, en contraposición al 70\% de hace 20 años (Freire, 2012).

Ante esta realidad — fruto de nuestro contexto social—, diversos estudios aluden a la existencia de un nuevo tipo de infancia (Karsten, 2005) que se caracteriza por pasar menos tiempo en contacto con la naturaleza que en ningún otro momento de la historia. Es por ello que las generaciones actuales presentan nuevas necesidades derivadas de la falta de vivencias de ocio en el exterior, una menor socialización en espacios públicos, un estilo de vida sedentario y una mayor relación con el mundo virtual que con el real, lo que incide negativamente en su desarrollo individual y como miembros de la sociedad. Pero, ¿cuáles son los motivos que originan esta realidad? La revisión de la literatura existente apunta a los procesos de antropización de los espacios (Cox, Shanahan, Hudson, Fuller \& Gaston, 2018), la proliferación de las tecnologías de la información y la comunicación (Zaradic \& Pergams, 2007), la excesiva preocupación de los progenitores por la seguridad de los hijos e hijas (Clements, 2004), la percepción de los espacios naturales cercanos como inseguros (Larson et al., 2018), la sobrecarga de las 
agendas infantiles y juveniles (Honoré, 2010) y el predominio de valores como el individualismo, el consumismo y la competitividad (Freire, 2011). Estos son, entre otros, los responsables de los cambios sociales actuales y los condicionantes de esa falta de contacto con el aire libre y la naturaleza por parte de los más jóvenes.

Si bien a priori esta realidad pudiera parecer poco relevante, lo cierto es que las investigaciones constatan que de esta ausencia de relación se derivan importantes consecuencias en una doble vertiente. Por un lado, para el bienestar en el sentido integral del término, esto es, físico (Louv, 2011), psicológico (Kaplan \& Kaplan, 1989; Louv, 2019) y social (Priego, 2011) y, por otro, para la propia conservación del medioambiente (Rosa \& Collado, 2019).

Precisamente, esta última idea cobra especial relevancia en un momento de la historia en el que la crisis ecológica constituye uno de los retos principales del planeta (Evans, 2019). Podrá parecernos casual o incluso anecdótico, pero lo cierto es que no resulta fruto de la coincidencia que dicha crisis comenzara a hacerse más evidente a medida que avanzaba nuestra desvinculación con la naturaleza (Programa de las Naciones Unidas para el Medio Ambiente, PNUMA, 2007), siendo esta "un síntoma de ruptura entre la relación emocional y espiritual del ser humano con el medio natural” (Kellert, 1993, p. 26). Ello porque, desde el momento en que el ser humano se recluyó en las ciudades y comenzó a sentir que la naturaleza no era imprescindible para su subsistencia —reduciéndola a mera facilitadora de recursos y materias primas-, comenzaron a hacerse claramente visibles los límites de nuestro planeta. Ante ello, la sociedad se vio obligada a tomar conciencia de que la naturaleza era mucho más que un espacio abastecedor de bienes infinitos y que, por tanto, el sistema de explotación al que estaba siendo expuesta era insostenible. De esta forma, a mediados del siglo pasado surgieron las primeras estrategias para tratar de cambiar de rumbo, siendo una de las más relevantes la educación ambiental.

\section{Marco teórico}

\section{La experiencia de ocio en la naturaleza: implicaciones para la educación ambiental}

Desde un planteamiento tradicional se ha considerado que los conocimientos ambientales son un factor determinante para promover comportamientos a favor del medioambiente (Durán, Alzate, López y Sabucedo, 2007; Sánchez y De la Garza, 2015), supeditando, por tanto, la acción a la dimensión cognitiva de las personas. Este enfoque lineal y de causa-efecto parte de la consideración de que un mayor conocimiento ambiental conduce a actitudes a favor del medio, las cuales a su vez implican un comportamiento específico de protección del entorno. Sin embargo, hace años se observó que esta correlación entre conocimientos, actitudes y conductas es baja por regla general, lo que nos permite concluir, por un lado, que un elevado conocimiento ambiental no implica necesariamente un comportamiento ecológico (Hungerford \& Volk, 1990; Steg \& Vlek, 2009) y, por otro, que tener actitudes positivas respecto del medio ambiente tampoco (Collado y Corraliza, 2016).

Así, sin negar la relevancia de los trabajos científicos que han demostrado que los conocimientos ambientales son un elemento relevante (Bradley, Waliczek \& Zajicek, 1999; Cheng \& Monroe, 2012), todo parece apuntar a que por sí mismos no conducen a actitudes y comportamientos proambientales (Barazarte, Neaman, Vallejo y García, 2014; Collado y Corraliza, 2016; Hungerford \& Volk, 1990; Sánchez y De la Garza, 2015), especialmente en el caso de la infancia (Sobel, 2013) y que el proceder humano es mucho más complejo y está influenciado por otros factores (Álvarez y Vega, 2009). El grado de conocimiento es únicamente un elemento más —y no parece ser el más relevante- en el desarrollo de comportamientos proambientales (Corraliza y Collado, 2019).

Por ello, en la literatura científica surgen otros modelos que incluyen diversas variables personales y situacionales consideradas como influyentes en las conductas ecológicas. Sin embargo, muchos de ellos tienen en común que centran su atención en los factores cognitivos, obviando la existencia de otra dimensión fundamental en el 
comportamiento humano: la emocional (Duerden \& Witt, 2010; Durán et al., 2007), porque es aquí donde tienen cabida los componentes afectivos. Por ejemplo, se ha constatado que las actitudes afectivas de las personas hacia la naturaleza desempeñan un papel importante en la toma de decisiones respecto de la conservación de la biodiversidad (De Pinho, Grilo, Boone, Galvin \& Snodgrass, 2014; Soga et al., 2016).

En este sentido, diferentes investigaciones se han centrado en los últimos años en esta dimensión de la persona como factor que promueve la sensibilidad ambiental, requisito previo para que se produzca un comportamiento proambiental (Hungerford \& Volk, 1990). Otros conceptos muy similares como afinidad emocional hacia la naturaleza (Kals, Schumacher \& Montada, 1999) o conexión con la naturaleza (Stephan Mayer \& McPherson Frantz, 2004) se han utilizado para definir el "sentido afectivo y experiencia de unidad con el mundo natural" (p. 504), aludiendo a la necesidad de que las personas se sientan conectadas con estos entornos para percibirse responsables de los mismos y, en consecuencia, se preocupen de su conservación y protección.

Desde esta óptica, autores como Corraliza y Collado (2019) ponen el acento en la importancia de que los programas de educación a mbiental contemplen la perspectiva afectiva como un elemento clave, ya que promueven la conexión emocional con el entorno. La mayoría de estos programas se basa fundamentalmente en la trasmisión de información acerca de cuestiones ambientales enfocándose, por tanto, en la esfera cognitiva de la persona (Rickinson, 2001). Incluso el propio planteamiento de la educación ambiental escolar tiende a centrarse en los contenidos en lugar de los aspectos emocionales (Freire, 2011; Sobel, 2013).

A tenor de lo expuesto, la revisión de la literatura pone de manifiesto que uno de los principales factores que parece contribuir a la conectividad con la naturaleza es la frecuencia de contacto y disfrute con el mundo natural durante la infancia y la adolescencia (Chawla \& Derr, 2012; Cheng \& Monroe, 2012; Louv, 2018; Wells $\&$ Lekies, 2006). Por ello, y partiendo de esta premisa, es evidente que la progresiva desvinculación de los más jóvenes con los entornos verdes guarda una estrecha relación con las actitudes y comportamientos proambientales (Evans, Otto \& Kaiser, 2018), de tal forma que el distanciamiento social con la naturaleza podría traer consigo consecuencias negativas para el medioambiente (Evans, 2019).

Es por ello que si la literatura científica ha demostrado que los conocimientos teóricos no son suficientes por sí mismos para desarrollar comportamientos proambientales y que, para lograrlos, es necesario educar también la parte afectiva y emocional de las personas, debemos encontrar herramientas que nos ayuden en esta tarea. En este sentido, el ocio se nos presenta como un recurso de extraordinaria potencialidad. En sintonía con lo expuesto en la Carta Internacional para la Educación del Ocio (World, Leisure and Recreation, WLRA, 1994), o con lo defendido por Cuenca (2014), entendemos el ocio como una experiencia humana compleja, íntimamente relacionada con la autonomía responsable, la satisfacción, el disfrute y la felicidad. Un recurso valioso para el desarrollo personal y comunitario (Maroñas, Martínez y Gradaílle, 2019), con importantes repercusiones en el bienestar y la calidad de vida no solo de los humanos, sino del planeta, pues también se ven afectados — positiva o negativamente- los contextos en los que se llevan a cabo dichas vivencias (Arruti, 2011).

Especialmente interesante para el tema que nos ocupa es la denominada dimensión ambiental-ecológica del ocio (Cuenca, 2014), pues pone el acento no tanto en la experiencia (lúdica, solidaria, festiva o creativa), sino en el contexto en el que se realiza. Desde esta perspectiva, tener vivencias de ocio en la naturaleza supone contar con ventajas que no se dan en otro tipo de ambientes artificiales, pues "los espacios naturales contienen cualidades propias, diríamos ontológicas, que los presentan como espacios privilegiados para el ocio" (Ried, 2015, p. 506). Cabe destacar, además, que estas ventajas transitan más allá de los beneficios para el bienestar humano (físico, psicológico y social) para abarcar también el despertar de la conciencia ambiental (afinidad emocional con la naturaleza). No obstante, es necesario resaltar que dichas experiencias de ocio siempre deben tener como punto de partida y horizonte la sostenibilidad, muy especialmente cuando tienen lugar en entornos protegidos. Con ello aludimos al 
desarrollo de lo que se conoce como ocio sostenible (Tirone \& Halpenny, 2017), esto es, un ocio impregnado no solo de valores de disfrute, goce y bienestar, sino que a su vez asu me los principios de "responsabilidad compartida, de sostenibilidad, de protección y de conservación de la naturaleza” (Arruti, 1996, p. 93).

Sobre la base de estos planteamientos teóricos, el presente artículo analiza el grado de conexión emocional con la naturaleza de una muestra de adolescentes de la provincia de Pontevedra ${ }^{1}$ y su relación con sus experiencias de ocio en entornos naturales. Asimismo, estudiamos la influencia de otros factores en dicha conexión, como el lugar de residencia y el género.

\section{Metodología}

El presente trabajo se enmarca en el enfoque cuantitativo, siendo la técnica de encuesta la seleccionada y el cuestionario el instrumento escogido. A continuación se presenta dicho instrumento y su proceso de validación, así como los ítems con los que se trabajó en la investigación, la población objeto de estudio y la selección de la muestra.

\section{Instrumento y proceso de validación}

El instrumento de recogida de datos fue un cuestionario elaborado ad hoc, sometido a una exhaustiva evaluación para analizar su validez de contenido. Para ello, se recurrió al juicio de expertos, concretamente el método de agregados individuales (Corral, 2009). En total se seleccionaron 10 jueces que ofrecieron una valoración, tanto cualitativa como cuantitativa, del cuestionario. En este último caso se analizó estadísticamente la pertinencia y la precisión de los ítems.

En lo referido a la pertinencia, se calculó el índice de acuerdo entre los jueces, siendo el grado de concordancia el que nos indicaría la confiabilidad de los juicios y, por ende, la validez de los mismos. Para ello se recurrió al modelo de Lawshe (1975), modificado por Tristán-López (2008), que consiste en la evaluación independiente de cada uno de los ítems sobre la base de tres posibilidades: esencial, útil pero no esencial e innecesario.

Una vez que los jueces valoraron cada ítem en consonancia con esta triple escala se determinó el número de coincidencias de la valoración "esencial", buscando un amplio acuerdo entre ellos. Para establecer dicho consenso Lawshe (1975) propone la razón de validez de contenido (CVR). Así, tras aplicar el modelo modificado por Tristán-López $(2008)^{2}$ todos los ítems alcanzaron la validez de contenido mínima exigible $(0,58)$. En cuanto al valor de validez global del instrumento se obtuvo un puntaje de 0,94 (o 94\%), por lo que podemos concluir que el instrumento, en su conjunto, es pertinente pues superó con amplitud el mínimo de 0,58.

1. Pontevedra es una de las cuatro provincias de la Comunidad Autónoma de Galicia (España). Tiene 942.665 habitantes (Instituto Galego de Estatística, 2019), de los que algo más del 40\% se concentran en las ciudades de Vigo (295.364 habitantes, la más poblada de Galicia) y Pontevedra (83.029 habitantes). Cuenta con un territorio de $4.495 \mathrm{~km} 2$ $y$, tanto en el litoral como en el interior de la provincia encontramos numerosas zonas reconocidas como espacios naturales con diferente grado de protección.

2. El modelo de Lawshe (1975) adolece de dos problemáticas fundamentales: a) que no está previsto para menos de cinco jueces y b) que los valores mínimos exigidos para CVR se ven afectados por el número de jueces, siendo muy exigente cuando se cuenta con pocos (CVR $=0,99$ con cinco jueces) y excesivamente laxo cuando hay un número amplio de ellos (CVR = 0,29 para 40 jueces) (Tristán-López, 2008). Para solventar esta problemática se planteó la modificación propuesta por Tristán-López (2008). Este autor, sobre la base de una corrección del valor mínimo de CVR establecido por Lawshe (1975), normalizó la población de jueces logrando que la alternativa de CVR, es decir, CVR', sea constante, independientemente del número de jueces, con lo cual no solamente se resuelve el problema del efecto de tamaño, sino también la interpretación de los acuerdos en la relación de validez de contenido. Como CVR' no depende de N, cuando $\mathrm{N}$ tiende a 8, CVR' permanece constante en 0,5823 , lo cual conduce a una condición de igual exigencia en todos los casos, independientemente del número de jueces (Tristán-López, 2008). 
En cuanto a la precisión de cada ítem, para su análisis estadístico procedimos, al igual que otros autores (véase, por ejemplo, Sanz-Arazuri, Alonso-Ruíz, Valdemoros-San-Emeterio y Ponce-de-León-Elizondo, 2013) al cálculo de descriptivos. Así, establecimos la media y la desviación típica de las valoraciones de cada juez para cada uno de los ítems. Estas podían oscilar entre $1=$ nada preciso y $4=$ muy preciso. A partir de ello se determinó un punto de corte mínimo que se estableció en tres puntos (bastante preciso) para no tener que recurrir obligatoriamente a su reformulación. Tras el análisis se constató que la totalidad de los ítems alcanzaron el mínimo de corte establecido (la puntuación más baja fue de 3,40) y que el instrumento en su conjunto arrojó una puntuación media de 3,81, pudiendo considerarse, por tanto, que el instrumento era preciso.

Asimismo, también se tuvieron en cuenta las sugerencias cualitativas aportadas por los jueces y, con el objeto de testear su funcionalidad, el cuestionario fue sometido a una prueba piloto o pretest que permitió garantizar su calidad y funcionamiento.

\section{Ítems objeto de estudio}

Tras la validación del instrumento se procedió al diseño final, con lo cual este quedó conformado por cinco bloques. En el presente artículo se presentan los ítems de dos de ellos:

- El primer bloque, denominado "datos de identificación", del que se tomaron los relativos al género (chico y chica) y al lugar habitual de residencia (aldea, pueblo y ciudad).

- El tercer bloque, denominado "ocio y contacto con la naturaleza", del cual se analizaron las preguntas 7 y 8 , concretamente los ítems 7.1, 7.2 y 8.1, 8.2. En ellas se solicitó que el alumnado identificara la frecuencia con la que, en los últimos 12 meses, había realizado determinadas actividades de ocio en contacto directo, indirecto y vicario con la naturaleza. Para ello, se propuso una escala Likert de cinco niveles: nunca, casi nunca, algunas veces, casi siempre y siempre. La única diferencia entre ambas preguntas es que la 7 se centraba en los días lectivos (lunes a viernes) y la 8 en los no lectivos (sábado y domingo). De forma específica los ítems 7.1 y 8.1 aludían a la frecuencia de disfrutar de alguna experiencia de ocio directa en un espacio natural (campo, bosque, montańa, río, etc.), en días lectivos y no lectivos respectivamente, mientras que los ítems 7.2 y 8.2 hacían referencia a la frecuencia de ir a ver y/o fotografiar animales y/o paisajes al aire libre, también los días lectivos y no lectivos.

Asimismo, en este bloque se recurrió a la pregunta 9, una escala de conexión emocional con el medio natural. Para su elaboración se tomó como referencia el trabajo de Cheng y Monroe (2012) que presenta un índice de conectividad con la naturaleza para la infancia. No obstante, no se utilizó dicho índice — pues se centra en la etapa infantil - sino que a partir de los pasos que siguieron los autores para su construcción, se realizó una adaptación que fue sometida a un análisis de fiabilidad y consistencia interna. Para ello, se recurrió al coeficiente alfa de Cronbach ( $\alpha$ ), tomando como valor mínimo exigido 0,70, tal y como sugieren algunos autores, por ejemplo, Hernández, Fernández y Baptista (2014). Finalmente nuestra escala alcanzó un puntaje de 0,798 quedando, por tanto, garantizada su fiabilidad.

La escala estuvo integrada por 10 ítems con cuatro intervalos de respuesta (muy en desacuerdo, en desacuerdo, de acuerdo, y muy de acuerdo). Tras la aplicación del cuestionario, dicha escala se tradujo en puntuaciones numéricas, de tal forma que se pudiera calcular la media de conexión emocional con la naturaleza. Se otorgaron las siguientes puntuaciones: muy en desacuerdo $=1$ punto; en desacuerdo $=2$; de acuerdo $=3$; y muy de acuerdo = 4 puntos. Por tanto, el valor máximo serían 40 puntos y el mínimo 10. Tras obtener las puntuaciones medias de todo el alumnado se aplicaron los siguientes rangos para determinar el grado de conexión emocional: de 1 a 2 puntos = conexión nula o muy baja; de 2,1 a 2,5 = baja; de 2,6 a 3 = neutra; de 3,1 a 3,5 = alta; y de 3,6 a $4=$ muy alta. Para establecer dichos rangos se empleó el mencionado trabajo de Cheng y Monroe (2012). 


\section{Población y muestra}

Tras la validación del cuestionario, este se aplicó a una muestra de estudiantes de educación secundaria obligatoria y posobligatoria (bachillerato) de la provincia de Pontevedra. Una vez que se tomó como referencia el tamaño de la población en el curso académico 2016-2017 (46.857), un margen de error del 3,8\% y un nivel de confianza del $95 \%$, dado el supuesto de $\mathrm{p}=\mathrm{q}=0,5$, se debía cubrir una muestra mínima de 652 sujetos. Para su consecución se optó por un muestreo no probabilístico por cuotas cruzadas con afijación proporcional por cada uno de los niveles de estratificación establecidos que son los siguientes:

a) Grado de urbanidad de los ayuntamientos de la provincia de Pontevedra determinado por el Instituto Galego de Estatística (IGE) para el año 2016 (Zona Densamente Poblada: zDp; Zona Intermedia Poblada: ZIP; y Zona Poco Poblada: ZPP).

b) Etapa educativa (educación secundaria obligatoria y bachillerato). En ambas etapas se trabajó con los cursos impares; esto es: $1^{\circ}$ de eso, $3^{\circ}$ de eso y $1^{\circ}$ de BAC.

Respecto de esto último, no se contempló aplicar el cuestionario en todos los cursos para evitar la sobrecarga de los centros a la hora de solicitar su colaboración, sumado a la dificultad de acceso al alumnado de $2^{\circ}$ de BAC. No obstante, se considera que con esta selección se obtenía una adecuada panorámica de ambas etapas educativas, pues en las clases había alumnado de todas las edades (de 11-12 años a 17-18 años). Igualmente, y a fin de disminuir lo máximo posible los sesgos, la elección de las aulas en cada centro se realizó aleatoriamente siempre que fue posible. Además, se seleccionaron centros educativos en función de la distribución Arco AtlánticoArco Interior, para garantizar la presencia de escolares procedentes de la costa y del interior, así como centros públicos y privados/concertados, con lo cual se respetaba la proporción de la Comunidad Autónoma de Galicia (aproximadamente, de cada tres centros, dos son públicos).

Tras el trabajo de campo — que atendió a todos los criterios expuestos_-, se obtuvo la muestra definitiva que resultó ser algo superior (683 sujetos) a la muestra teórica establecida (652). Con ello el error muestral fue ligeramente inferior al de partida (3,7\% vs 3,8\%). En este sentido, se estimó que se asemejaba a lo planteado, guardando una adecuada proporcionalidad, por lo que no se aplicó ningún factor de ponderación para adaptar los pequeños desajustes.

La aplicación de los cuestionarios se realizó en 2017 y estuvo guiada en el aula durante una sesión de clase, para garantizar en todo momento el anonimato del alumnado participante.

Por su parte, para el análisis estadístico de los datos se empleó el spss Statistics, versión 22 para Windows (2013). En cuanto a las pruebas empleadas se recurrió a las de tipo no paramétrico. Concretamente para la comparación entre muestras independientes (chicos/chicas) se acudió a la prueba U de Mann-Whitney; para la comparación entre tres muestras independientes (aldea/pueblo/ciudad) se recurrió a la prueba de Kruskal-Wallis; y para el grado de asociación lineal (positiva y negativa) entre dos variables se empleó el coeficiente de correlación de Spearman.

\section{Resultados y discusión}

Comenzamos analizando cómo estaban de conectados los adolescentes con la naturaleza sobre la base de la escala anteriormente explicada (Figura 1). Tal como se puede observar, los porcentajes más elevados se sitúan en un grado que identificamos como neutro $(36,9 \%)$ - ni conectado ni desconectado-y alto (35,5\%). Aquellos que obtuvieron una conexión muy alta apenas superaron la décima parte de la muestra (11,1\%), mientras que aquellos cuya manifestación era muy baja o baja alcanzaron el 16,5\%. Así, se observa que una parte considerable de la muestra tuvo una conexión emocional alta o muy alta con la naturaleza, aunque también fue elevado el 
porcentaje de los que se situaron en una posición neutra. Aquellos cuya conexión era baja o muy baja fueron minoritarios, si bien tampoco es un porcentaje despreciable, lo que supone considerar esta situación, pues son múltiples los trabajos que sugieren que la conexión emocional con el medio natural está relacionada con las actitudes y comportamientos proambientales (Cheng \& Monroe, 2012; Durón-Ramos, Collado, García-Vázquez \& Bello-Echevarría, 2020; Otto \& Pensini, 2017; Whitburn, Linklater \& Abrahamse, 2019).

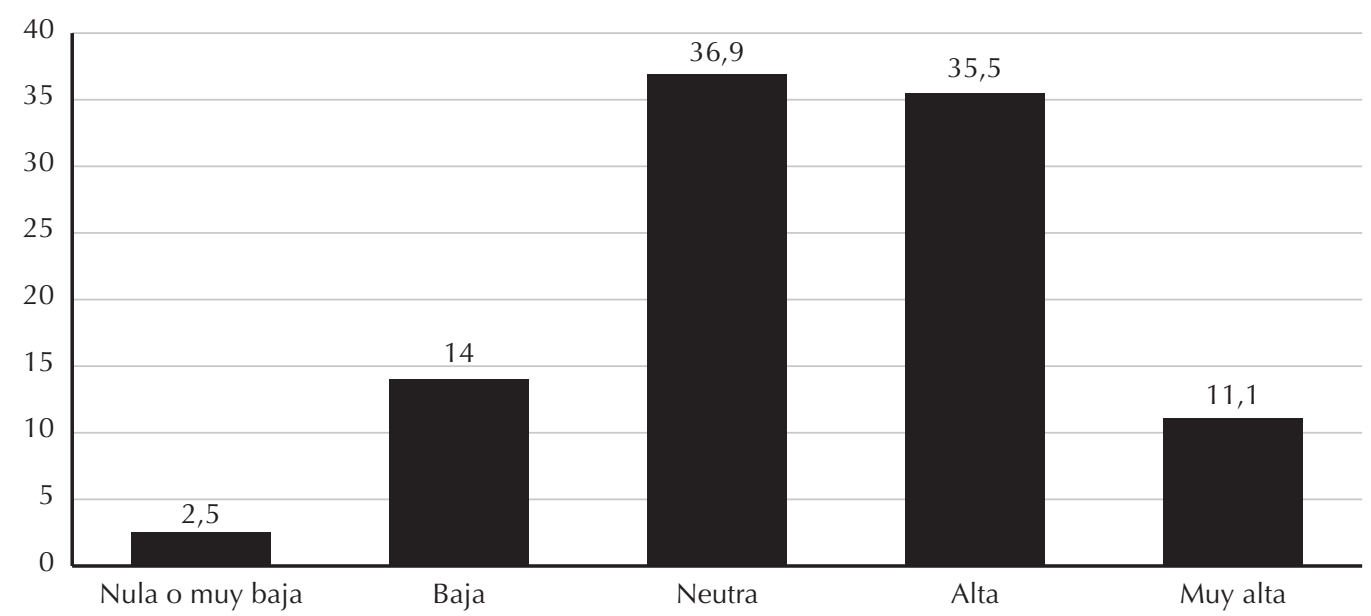

Figura 1. Grado de conexión emocional de los adolescentes con la naturaleza (\%).

Fuente: Elaboración propia.

Desde esta óptica, uno de los factores que parece tener mayor influencia en la conexión emocional con la naturaleza es su disfrute a través de vivencias gratificantes (Cagle, 2018; Gray \& Pygott, 2018; Kals et al., 1999; Rosa, Cabicieri \& Collado, 2018). Por ello estudiamos si la frecuencia de experiencias de ocio directas en la naturaleza por parte de los adolescentes encuestados se relacionaba con su grado de conexión emocional. Para ello aplicamos el coeficiente de correlación de Spearman (Tabla 1).

Tabla 1

Correlación entre experiencias de ocio directas en contacto con la naturaleza y el grado de conexión emocional con la misma ( $r$ de Spearman)

Frecuencia de experiencias directas de contacto con la naturaleza

Grado de conexión emocional con la naturaleza

\begin{tabular}{lll}
\multicolumn{2}{c}{ contacto con la naturaleza } & $\mathrm{R}$ \\
\hline \multirow{2}{*}{$\begin{array}{l}\text { Pasar tiempo de ocio en espacios } \\
\text { naturales (campos, bosques, etc.). }\end{array}$} & Entre semana & $0,295^{* * *}$ \\
\cline { 2 - 3 } $\begin{array}{l}\text { Ir a ver y/o fotografiar animales, } \\
\text { paisajes al aire libre }\end{array}$ & Enes de semana & $0,414^{* * *}$ \\
\cline { 2 - 3 }${ }_{* * *}<<0,001$. & Fines de semana & $0,319^{* * *}$ \\
\hline
\end{tabular}

Fuente: Elaboración propia.

A partir de los datos se desprende que ambas experiencias de contacto directo, tanto entre semana como los fines de semana, presentaron una correlación positiva altamente significativa $(p<0,001)$ con el grado de conexión emocional con la naturaleza: a medida que aumentaba la frecuencia de experiencias directas en el medio natural, 
aumentó también el grado de conectividad. Por tanto, cuanto mayor era el tiempo de ocio que pasaban los adolescentes vinculados con este tipo de entornos, mayor era su conexión con los mismos. Estos datos apoyan los de otros estudios que han constatado esta misma tendencia (Cheng \& Monroe, 2012; Collado y Corraliza, 2016; Durón-Ramos et al., 2020; Giusti, Svane, Raymond \& Beery, 2018; Lumber, Richardson \& Sheffield, 2017), poniendo de manifiesto la importancia que alcanzan las vivencias de ocio en la naturaleza desde una perspectiva ambiental (Ried, 2015; Rosa \& Collado, 2019).

Igualmente, si bien se revela que los adolescentes encuestados están relativamente conectados con la naturaleza, recordemos que existe un 16,4\% que manifestó una muy baja o baja conexión emocional. Se trata, por tanto, de un grupo de jóvenes que no presenta interés alguno por el medio natural y carece de vinculaciones con el mismo, pudiendo ser personas que presentan rasgos biofóbicos. Para comprobarlo, analizamos el ítem específico de la escala que aludía a la consideración de la naturaleza como espacio incómodo y/o desagradable (Figura 2).

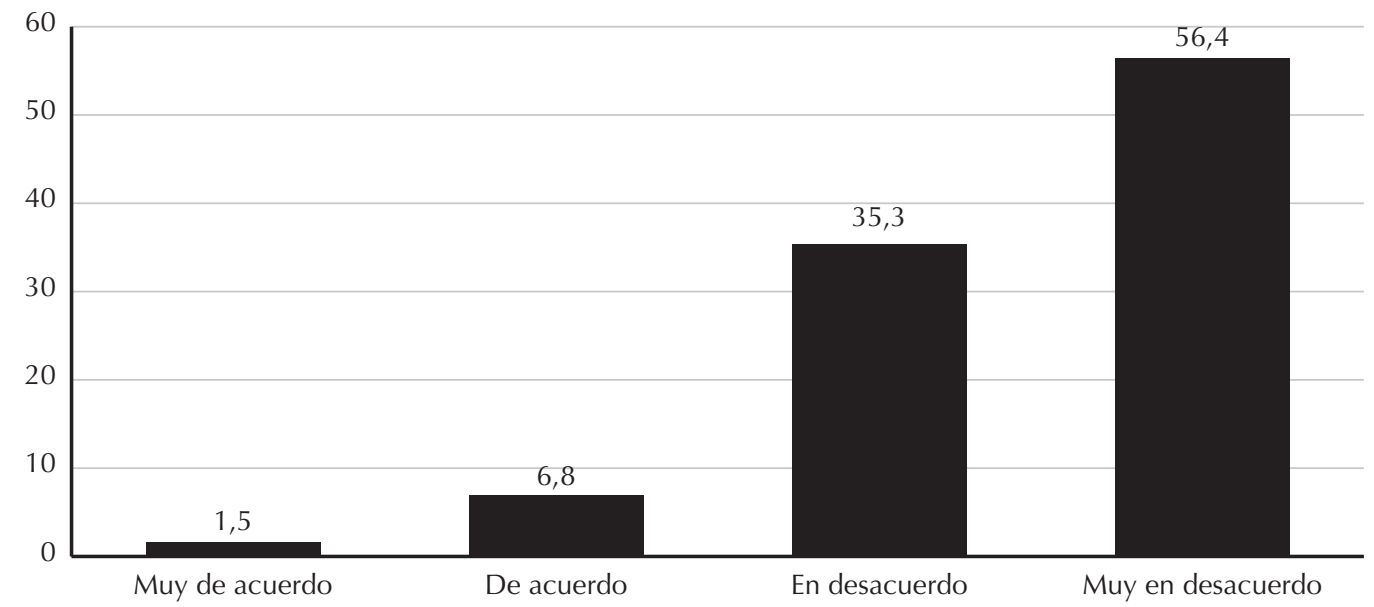

Figura 2. Grado de acuerdo con la afirmación "estar en entornos naturales es incómodo y/o desagradable" (\%).

Fuente: Elaboración propia.

Tal como muestra la Figura 2, la gran mayoría de los chicos y chicas encuestados se manifestó muy en desacuerdo o en desacuerdo (91,7\%) con esta consideración, por lo que apenas uno de cada 10 adolescentes presentaría rasgos biofóbicos. Esta tendencia refuerza las conclusiones de múltiples estudios que afirman que los seres humanos, en términos generales, presentan un interés e inclinación innata hacia la naturaleza (Herzog, Maguire \& Nebel, 2003; Ulrich, 1993) aludiendo, por un lado, a la hipótesis de la biofilia (Kellert \& Wilson, 1993) y, por otro, a las cualidades restauradoras con las que cuentan estos entornos y que impulsan la atracción humana hacia los mismos (Kaplan \& Kaplan, 1989; Collado y Corraliza, 2016). Sin embargo, la literatura revela que esta tendencia instintiva suele ser baja y susceptible de ser modelada por el aprendizaje y la cultura, al forjar sentimientos que pueden ir desde la atracción y la indiferencia hasta la aversión; esto es, se puede transitar de sentimientos biofílicos a sentimientos biofóbicos (Kellert, 1993).

Con estos datos resulta de interés analizar si el rasgo biofóbico que hemos estudiado se asociaba a una menor conexión emocional hacia la naturaleza. Para ello se aplicó el coeficiente de correlación de Spearman, con lo cual se confirmó la existencia de una asociación negativa altamente significativa $(r=-0,350 ; p<0,001)$. Esto es, cuanto más de acuerdo se estaba en considerar la naturaleza como un entorno incómodo y/o desagradable menor 
era el grado de conectividad que se tenía con ella. Por tanto, los sentimientos biofóbicos dificultan vincularse al medio natural, con las consecuencias que ello implica en clave proambiental, ya que las personas con aversión a la naturaleza se benefician igualmente de sus recursos, sin llevar a cabo acciones que la protejan (Hueso, 2017).

Por ello - y partiendo de la base de que una mayor frecuencia de disfrute y contacto con la naturaleza se vincula con una mayor conexión, tal y como observamos anteriormente-, parecería razonable considerar el lugar de residencia de los adolescentes encuestados como un factor influyente en su grado de conexión, pues las posibilidades de vivencias directas con la naturaleza no son las mismas en las ciudades que en el campo. De hecho, los entornos urbanos son espacios en los que la relación con zonas verdes está mucho más limitada en comparación con los contextos rurales (Gifford \& Nilson, 2014; Priego, 2011). Desde esta perspectiva, se aplicó la prueba de Kruskal-Wallis y, efectivamente, se confirmó la existencia de diferencias altamente significativas (Tabla 2).

Tabla 2

Comparación entre el grado de conexión emocional con la naturaleza y el lugar de residencia (prueba de Kruskal Wallis)

\begin{tabular}{|c|c|c|c|c|}
\hline $\begin{array}{c}\text { Conexión emocional con } \\
\text { la naturaleza }\end{array}$ & $\begin{array}{l}\text { Lugar de } \\
\text { residencia }\end{array}$ & $\mathrm{N}$ & $\begin{array}{l}\text { Rango } \\
\text { promedio }\end{array}$ & $X^{2}$ \\
\hline \multirow{3}{*}{$\begin{array}{l}\text { Grado de conexión } \\
\text { emocional con la } \\
\text { naturaleza }\end{array}$} & Aldea & 185 & 383,42 & \multirow{3}{*}{$15,740^{* * *}$} \\
\hline & Pueblo & 273 & 320,00 & \\
\hline & Ciudad & 216 & 320,29 & \\
\hline
\end{tabular}

En la comparativa entre pares de categorías la prueba $U$ de Mann-Whitney confirmó que existían diferencias significativas entre aldea y pueblo $(Z=-3,549 ; p<0,001)$ y aldea y ciudad $(Z=-3,476 ; p<0,01)$ revelándose, según los rangos promedio, que el grado de conexión emocional era significativamente mayor en los adolescentes que habitaban en zonas rurales que en zonas urbanas o suburbanas, lo que concuerda con lo constatado por otros investigadores (Durón-Ramos et al., 2020; Hinds \& Sparks, 2008). Ello permite inferir que vivir en un entorno que ofrece mayores oportunidades de contacto directo con la naturaleza contribuye a que las personas tengan experiencias de este tipo con más frecuencia lo que, a su vez, favorece una mayor conexión.

Sin embargo, otros autores como Müller, Kals y Pansa (2009) no han encontrado estas diferencias significativas e incluso se ha observado la situación contraria (Broom, 2017), aludiendo a que, a pesar de las oportunidades que ofrece el entorno rural, la incidencia de factores como la relación que las personas establezcan con la naturaleza, puede marcar la diferencia (Collado y Corraliza, 2016). Parece ser que las experiencias que generan afinidad deben ser positivas y estar relacionadas con el disfrute (Evans et al., 2018), lo que no siempre sucede en los hábitats de tipo rural donde es habitual que el contacto se asocie con cuestiones laborales.

Mención aparte merece la variable género. Tras aplicar la prueba U de Mann-Whitney se constató que existían diferencias altamente significativas entre chicos y chicas $(Z=-5,119 ; p<0,001)$, donde ellas mostraron mayor conectividad emocional hacia el medio natural en comparación a ellos (Figura 3). Esta circunstancia también se ha observado en otros trabajos (Broom, 2017; Duarte, Escario \& Sanagustín, 2017; Durón-Ramos et al., 2020; Müller et al., 2009), lo que supone un aporte más a la literatura que aborda las diferencias de género en relación con la proambientalidad. 


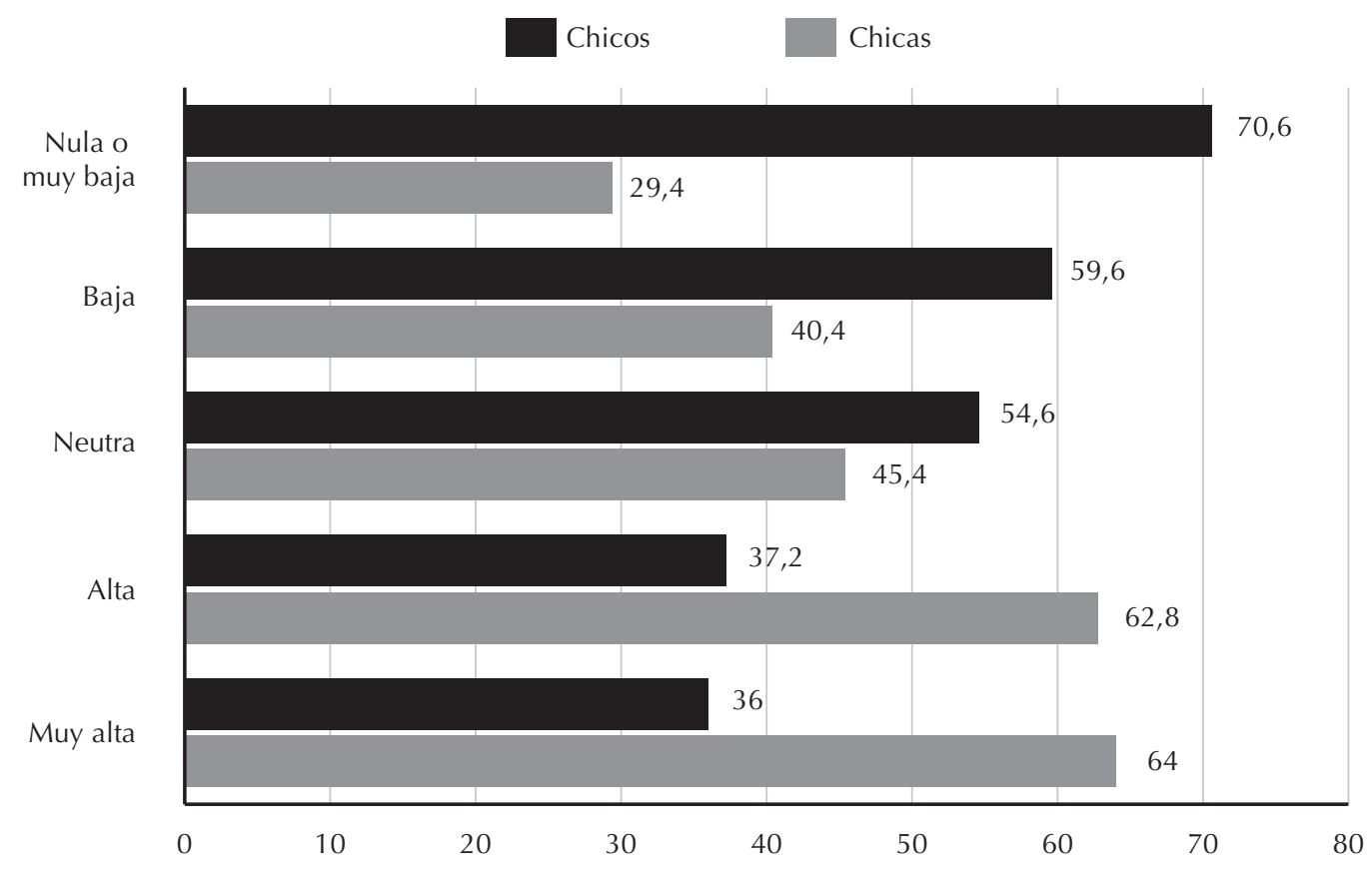

Figura 3. Comparación entre el grado de conexión emocional con la naturaleza y el género (\%).

Fuente: Elaboración propia.

Los motivos que podrían justificar esta realidad no están claros (Durón-Ramos et al., 2020). No obstante, algunos autores como McCright (2010) sugieren que se podría encontrar una explicación en la socialización de género, pues los valores y expectativas sociales conferidos a niños y niñas son diferentes, siendo ellas las que suelen ser educadas en mayor medida en el altruismo, el apego, la empatía, y la preocupación por los demás, incluido también el medioambiente.

\section{Conclusiones}

En los últimos decenios se ha producido una transformación sociocultural en lo que se refiere a la ocupación del tiempo de ocio del colectivo infanto-juvenil, un cambio en el que los espacios abiertos están siendo progresivamente substituidos por los cerrados. Los entornos están cada vez más urbanizados, las ciudades son cada día más grandes, y la libertad y autonomía de la infancia y la adolescencia se encuentran muy limitadas como consecuencia del miedo parental a la inseguridad. Además, las experiencias de juego libre son remplazadas por actividades sedentarias y, progresivamente, las experiencias primarias con el mundo real, con la naturaleza, han sido sustituidas por otras vicarias, muchas de ellas procedentes de los múltiples aparatos electrónicos, obviando que el mundo natural es el contexto de estimulación sensorial más poderoso.

Esta circunstancia es especialmente notoria en los contextos urbanos, donde las oportunidades de vivir un ocio vinculado con la naturaleza están mucho más limitadas que en los entornos rurales. Además, en estos últimos suelen perdurar este tipo de experiencias dada la mayor presencia de espacios abiertos y desestructurados; aunque también porque se mantiene una cultura más tradicional del juego, que convive con el ocio digital.

Si bien a priori esta nueva realidad social pudiera no parecer tan relevante, las consecuencias en clave de bienestar humano y medioambiental ya han sido documentadas en la literatura. De hecho, y en relación con las segundas, en este trabajo se ha constatado cómo un mayor tiempo de ocio en entornos naturales ha correlacionado de 
forma altamente significativa con una mayor conexión emocional con la naturaleza, lo que pone de manifiesto la importancia de que los más jóvenes disfruten de experiencias de ocio en espacios verdes debido a los beneficios que ello genera desde una perspectiva ambiental.

Desde esta óptica, los planteamientos de la educación ambiental, escolar y no escolar, deberían tomar como uno de sus ejes fundamentales los componentes emocionales y afectivos de las personas, especialmente durante la infancia para a partir de ellos y en edades adecuadas, comenzar a trabajar problemáticas ambientales en las que se puedan implicar cuestiones más cognitivas (perspectiva intelectual). En este planteamiento — que combina el binomio emoción-razón - el entorno natural cuenta con un gran potencial educativo, ya que ofrece diversas oportunidades para la exploración y el juego, lo cual es reconocido como "el contexto para el aprendizaje y no solo el foco del aprendizaje” (Kelly \& White, 2013, p. 6). Por tanto, lo ideal sería promover una educación ambiental que tenga lugar en el propio entorno (Palmer, 1998), es decir, una educación ambiental al aire libre.

En el logro de esta meta, la educación del ocio y más concretamente la educación de la dimensión ecológica del ocio, se presenta como una aliada a tener en cuenta, pues facilita el desarrollo de intereses, valores y aficiones que pueden contribuir a generar experiencias gratificantes en entornos naturales favorecedores de una conexión emocional con el medio. En esta tarea encuentran un desafío los centros educativos, las familias, el tejido asociativo, las instituciones comunitarias, etc.; ya que todas ellas han de facilitar tiempos y condiciones para que la infancia y la adolescencia pueda disfrutar responsablemente de experiencias de ocio valioso en la naturaleza.

No obstante, cabe destacar que a pesar de que el contacto con el medio natural parece ejercer influencia en la proambientalidad, los procesos que se encuentran detrás de esta relación no están claros (Wells \& Lekies, 2006). Se tiene constancia que, de alguna forma, este contacto - especialmente el libre, desestructurado y de disfrute-, ejerce influencia aunque no se sabe con certeza de qué forma y hasta qué punto. A pesar de ello, uno de los elementos que parece jugar un importante papel como mediador es el relativo a la conexión emocional con la naturaleza (Rosa et al., 2018) la cual, como se ha ido demostrando a lo largo de estas páginas, guarda una estrecha relación con el disfrute de los entornos naturales. A tenor de ello, el presente trabajo supone un aporte más a la literatura acerca de la relación entre el ocio en el medio natural y la conexión emocional hacia la naturaleza.

Con estas evidencias, creemos que está lejos de toda duda la importancia de que la población en general y el colectivo infantojuvenil en particular restituyan en sus vivencias de ocio cotidiano la vinculación con los espacios al aire libre y los entornos naturales, pues no solo contribuiremos a su bienestar, sino que estaremos apostando por una sociedad más comprometida con la búsqueda de soluciones a la crisis ecológica global.

El artículo original fue recibido el 10 de mayo de 2020

El artículo revisado fue recibido el 10 de mayo de 2020

El artículo fue aceptado el 12 de agosto de 2020

\section{Referencias}

Álvarez, P. y Vega, P. (2009). Actitudes ambientales y conductas sostenibles. Implicaciones para la educación ambiental. Revista de Psicodidáctica, 14(2), 245-260. Recuperado de https://www.redalyc.org/pdf/175/17512724006.pdf

Arruti, M. A. (1996). Ocio y naturaleza. Educación de la dimensión ecológica del ocio. Ciencias de la Educación, $156,87-96$.

Arruti, M. A. (2011). Educación del ocio y espacio natural protegido: una combinación ideal en la que los valores ocupan un lugar primordial. En A. Madariaga y J. Cuenca (Eds.), Los valores del ocio: cambio, choque e innovación (pp. 217- 239). Bilbao: Universidad de Deusto. 
Barazarte, R., Neaman, A., Vallejo, F., y García, P. (2014). El conocimiento ambiental y el comportamiento proambiental de los estudiantes de la enseñanza media, en la Región de Valparaíso (Chile). Revista de Educación, 364, 66-93. Recuperado de https://www.educacionyfp.gob.es/dctm/revista-de-educacion/articulos364/051. el-conocimiento-ambientalrev.ed.364.pdf?documentId=0901e72b818ff46f

Bradley, J. C., Waliczek, T. M., \& Zajicek, J. M. (1999). Relationship between environmental knowledge and environmental attitude of high school students. The Journal of Environmental Education, 30(3), 17-21. https://doi.org/10.1080/00958969909601873

Broom, C. (2017). Exploring the relations between childhood experiences in nature and young adults' environmental attitudes and behaviors. Australian Journal of Environmental Education, 33(1), 34-47. https://doi.org/10.1017/aee.2017.1

Cagle, N. L. (2018). Changes in experiences with nature through the lives of environmentally committed university faculty. Environmental Education Research, 24(1), 889-898. https://doi.org/10.1080/13504622.2017.1342116

Chawla, L. \& Derr, V. (2012). The development to conservation behaviors in childhood and youth. En S. Clayton (Ed.), Oxford handbook of environmental and conservation psychology (pp. 527-555). New York: Oxford University Press.

Cheng, J. C. \& Monroe, M. C. (2012). Connection to nature: Children's affective attitude toward nature. Environment and behavior, 44, 31-49. https://doi.org/10.1177/0013916510385082

Clements, R. (2004). An investigation of the status of outdoor play. Contemporary Issues in Early Childhood, 5(1), 68-80. https://doi.org/10.2304/ciec.2004.5.1.10

Collado, S. y Corraliza, J. A. (2016). Conciencia ecológica y bienestar en la infancia. Efectos de la relación con la naturaleza. Madrid: CCS.

Corral, Y. (2009). Validez y confiabilidad de los instrumentos de investigación para la recolección de datos. Revista Ciencias de la Educación, 19(33), 228-247. Recuperado de http://servicio.bc.uc.edu.ve/educacion/revista/n33/art12.pdf

Corraliza, J. A. y Collado, S. (2019). Conciencia ecológica y experiencia ambiental en la infancia. Papeles del Psicólogo, 40(2), 190-196. https://doi.org/10.23923/pap.psicol2019.2896

Cox, D. T. C., Shanahan, D. F., Hudson, H. L., Fuller, R. A., \& Gaston, K. J. (2018). The impact of urbanisation on nature dose and the implications for human health. Landscape and Urban Planning, 179, 72-80. https://doi.org/10.1016/j.landurbplan.2018.07.013

Cuenca, M. (2014). Ocio valioso. Bilbao: Universidad de Deusto.

De Pinho, J. R., Grilo, C., Boone, R. B., Galvin, K. A., \& Snodgrass, J. G. (2014). Influence of aesthetic appreciation of wildlife species on attitudes towards their conservation in Kenyan agropastoralist communities. PLoS ONE, 9(2), 1-10. https://doi.org/10.1371/journal.pone.0088842

Duarte, R., Escario, J. J., \& Sanagustín, M. V. (2017). The influence of the family, the school, and the group on the environmental attitudes of European students. Environmental Education Research, 23(1), 23-42. https://doi.org/10.1080/13504622.2015.1074660

Duerden, M. D. \& Witt, P. A. (2010). The impact of direct and indirect experiences on the development of environmental knowledge, attitudes, and behavior. Journal of Environmental Psychology, 30, 379-392. https://doi.org/10.1016/j.jenvp.2010.03.007

Durán, M., Alzate, M., López, W., y Sabucedo, J. M. (2007). Emociones y comportamiento pro-ambiental. Revista Latinoamericana de Psicología, 39(2), 287-296. Recuperado de https://www.redalyc.org/pdf/805/80539206.pdf

Durón-Ramos, M. F., Collado, S., García-Vázquez, F. I., \& Bello-Echevarría, M. (2020). The role of urban/rural environments on Mexican children's connection to nature and pro-environmental behavior. Frontiers in Psychology, 20, 1-6. https://doi.org/10.3389/fpsyg.2020.00514

Evans, G. W., Otto, S., \& Kaiser, F. G. (2018). Childhood origins of young adult environmental behavior. Psychological Science, 29(5), 679-687. https://doi.org/10.1177/0956797617741894

Evans, G. W. (2019). Projected behavioral impacts of global climate change. Annual Review Psychology, 70(1), 449-474. https://doi.org/10.1146/annurev-psych-010418-103023

Freire, H. (2011). Educar en verde. Ideas para acercar a niños/as a la naturaleza. Barcelona: Grao.

Freire, H. (2012). Una infancia saludable en la naturaleza. Ocio saludable, 4, 85-88.

Gifford, R. \& Nilsson, A. (2014). Personal and social factors that influence pro-environmental concern and behaviour: A review. International Journal of Psychology, 49(3), 141-157. https://doi.org/10.1002/ijop.12034 
Giusti, M., Svane, U., Raymond, Ch. M., \& Beery, T. H. (2018). A framework to assess where and how children connect to nature. Frontiers in Psychology, 8, 1-21. https://doi.org/10.3389/fpsyg.2017.02283

Gray, T. \& Pigott, F. (2018). Lasting lessons in outdoor learning: A facilitation model emerging from 30 years of reflective practice. Ecopsychology, 10(4), 195-204. https://doi.org/10.1089/eco.2018.0036

Henderson, K. A. \& Bialeschki, M. D. (2008). People and nature-based recreation. Leisure Sciences, 32(1), 1-2. https://doi.org/10.1080/01490400701881473

Hernández, R., Fernández, C., y Baptista, P. (2014). Metodología de la investigación. México, D.F.: McGrawHill.

Herzog, T., Maguire, C., \& Nebel, M. (2003). Assessing the restorative components of environments. Journal of Environmental Psychology, 28, 192-199. https://doi.org/10.1016/S0272-4944(02)00113-5

Hinds, J. \& Sparks, P. (2008). Engaging with the natural environment: The role of affective connection and identity. Journal of Environmental Psychology, 28(2), 109-120. https://doi.org/10.1016/j.jenvp.2007.11.001

Hofferth, S. L. \& Sandberg, J. F. (2001). Changes in American children's time, 1981-1997. En S. L. Hofferth \& T. J. Owens (Eds.), Children at the millennium: Where have we come from, where are we going? (pp. 1-7). New York: JAI.

Hofferth, S. L. \& Curtin, S. C. (2006). Changes in children's time, 19972002/3. An update. College Park: University of Maryland College Park.

Honoré, C. (2010). Bajo presión: cómo educar a nuestros hijos en un mundo hiperexigente. Madrid: RBA.

Hueso, K. (2017). Somos naturaleza: un viaje a nuestra esencia. Barcelona: Plataforma editorial.

Hungerford, H. R. \& Volk, T. L. (1990). Changing learner behavior through environmental education. Journal of Environmental Education, 21(3), 8-12. https://doi.org/10.1080/00958964.1990.10753743

Instituto Galego de Estatística (2019). Cifras oficiais da poboación a 1 de xaneiro. Ano 2019. Recuperado de https://bit.ly/3hVdv0Q

Kals, E., Schumacher, D., \& Montada, L. (1999). Emotional affinity toward nature as a motivational basis to protect nature. Environment and Behavior, 31(2), 178-202. https://doi.org/10.1177/00139169921972056

Kaplan, R. \& Kaplan, S. (1989). The experience of nature. New York: Cambridge University Press.

Karsten, L. (2005). It all used to be better? Different generations on continuity and change in urban children's daily use of space. Children's Geographies, 3(3), 275-290. https://doi.org/10.1080/14733280500352912

Kellert, S. (1993). The biological basis for human values of nature. En S. R. Kellert \& E. O. Wilson (Eds.), The biophilia hypothesis (pp. 42-69). New York: Island Press.

Kellert, S. \& Wilson, E. (1993). The biophilia hypothesis. New York: Island Press.

Kelly, J. \& White, E. J. (2013). The Ngahere Project: Teaching and learning possibilities in nature settings. New Zeeland: Wilf Malcolm Institute of Educational Research.

Larson, L. R., Szczytko, R., Bowers, E. P., Stephens, L. E., Stevenson, K. T., \& Floyd, M. F. (2018). Outdoor time, screen time, and connection to nature: troubling trends among rural youth? Environment and Behavior, 51(8), 966-991. https://doi.org/10.1177/0013916518806686

Lawshe, C. H. (1975). A quantitative approach to content validity. Personnel Psychology, 28, 563-575. https://doi.org/10.1111/j.1744-6570.1975.tb01393.x

Louv, R. (2011). Volver a la naturaleza. El valor del mundo natural para recuperar la salud individual y comunitaria. Barcelona: Integral.

Louv, R. (2018). Los últimos niños en los bosques: salvemos a nuestros hijos de trastorno por déficit de naturaleza. Madrid: Capitán Swing.

Louv, R. (2019). Naturaleza y salud. Barcelona: Gredos.

Lumber, R., Richardson, M., \& Sheffield, D. (2017). Beyond knowing nature: Contact, emotion, compassion, meaning, and beauty are pathways to nature connection. PLoS one, 12(5), 1-24. https://doi.org/10.1371/journal.pone.0177186

Maroñas, A., Martínez, R., y Gradaílle, R. (2019). Educación del ocio en y con la comunidad. Aportes desde la pedagogía social. Perfiles Educativos, 41(163), 111-126. https://doi.org/10.22201/iisue.24486167e.2019.163.58686

McCright, A. M. (2010). The effects of gender on climate change knowledge and concern in the American public. Population and Environment, 32, 66-87. https://dx.doi.org/10.1007/s11111-010-0113-1 
Müller, K., Kals, E., \& Pansa, R. (2009). Adolescents' emotional affinity toward nature: A cross-societal study. Journal of Development Process, 4(1), 59-69. Recuperado de http://citeseerx.ist.psu.edu/viewdoc/download?doi=10.1.1.465.963\&rep=rep1\&type=pdf

Otto, S. \& Pensini, P. (2017). Nature-based environmental education of children: Environmental knowledge and connectedness to nature, together, are related to ecological behaviour. Global Environmental Change, 47, 88-94. https://doi.org/10.1016/j.gloenvcha.2017.09.009

Palmer, J. A. (1998). Environmental education in the 21st century: Theory, practice, progress and promise. London: Routledge.

Programa de las Naciones Unidas para el Medio Ambiente, PNUMA (2007). Editorial. Tunza, 5(2), 3. Recuperado de https://es.slideshare.net/AndreaVillavicencio4/tunza-53-spanish

Priego, C. (2011). Naturaleza y sociedad. El valor de los espacios verdes urbanos. Madrid: Ministerio de Medio Ambiente y Medio Rural y Marino.

Ried, A. (2015). La experiencia de ocio al aire libre en contacto con la naturaleza como vivencia restauradora de la relación ser humano-naturaleza. Polis, Revista Latinoamericana, 14(41), 499-516. https://doi.org/10.4067/s0718-65682015000200029

Rickinson, M. (2001). Learners and learning in environmental education: A critical review of the evidence. Environmental Education Research, 7(3), 207-320. https://doi.org/10.1080/13504620120065230

Rosa, C. D., Cabicieri, C., \& Collado, S. (2018). Nature experiences and adults' self-reported pro-environmental behaviors: The role of connectedness to nature and childhood nature experiences. Frontiers in Psychology, 9, 1-10. https://doi.org/10.3389/fpsyg.2018.01055

Rosa, C. D. \& Collado, S. (2019). Experiences in nature and environmental attitudes and behaviors: Setting the ground for future research. Frontiers in Psychology, 10, 1-10. https://doi.org/10.3389/fpsyg.2019.00763

Sánchez, M. P. y De la Garza, A. (2015). Biofilia y emociones: su impacto en un curso de educación ambiental. RICSH, Revista Iberoamericana de las Ciencias Sociales y Humanisticas, 4(8), 1-9. https://doi.org/10.23913/ricsh.v4i8.42

Sanz-Arazuri, E., Alonoso-Ruíz, R. A., Valdemoros-San Emérito, M. Á., y Ponce-de-León-Elizondo, A. (2013). Validación de un cuestionario que analiza cómo trabaja el profesorado de la etapa infantil la educación para la salud desde el ámbito motor. Revista Iberoamericana de Diagnóstico y Evaluación, 1(35), 9-34. Recuperado de https:/www.redalyc.org/pdf/4596/459645435002.pdf

Sobel, D. (2013). Beyond ecophobia: Reclaiming the heart in nature education. Massachusetts: The Orion Society.

SPSS (versión 22) [Software]. (2013).

Soga, M., Gaston, K. J., Yamaura, Y., Kurisu, K., \& Hanaki, K. (2016). Both direct and vicarious experiences of nature affect children's willingness to conserve biodiversity. International Journal of Environmental Research and Public Health, 13(6), 1-12. https://doi.org/10.3390/ijerph13060529

Stephan Mayer, F. \& McPherson Frantz, C. (2004). The connectedness to nature scale: A measure of individual's feeling in community with nature. Journal of Environmental Psychology, 24(4), 503-515. https://doi.org/10.1016/j.jenvp.2004.10.001

Steg, L. \& Vleck, C. (2009). Encouraging pro-environmental behavior: An integrative review and research agenda. Journal of Environmental Psychology, 29, 1-9. Recuperado de https:/www.rug.nl/staff/e.m.steg/stegvlekencouraging.pdf

Tirone, S. \& Halpenny, E. (2017). Leisure and sustainability. Leisure/Loisir, 41(3), 277-280. https://doi.org/10.1080/14927713.2017.1368239

Tristán-López, A. (2008). Modificación al modelo de Lawshe para el dictamen cuantitativo de la validez de contenido de un instrumento objetivo. Avances en Medición, 6(1), 37-48. Recuperado de https:/www.semanticscholar.org/paper/Modificaci\%C3\%B3n-al-modelo-de-Lawshe-para-el-dictamenTrist\%C3\%A1n-L\%C3\%B3pez/401781bbf691f0c15965fcf667e98a48e4899165

Ulrich, R. S. (1993). Biophilia, biophobia and natural landscapes. En S. R. Kellert \& E. O. Wilson (Eds.), The biophilia hypothesis (pp. 73-177). New York: Island Press.

Wells, N. M. \& Lekies, K. (2006). Nature and the life course: Pathways from childhood nature experiences to adult environmentalism. Children, Youth and Environments, 16, 2-25. Recuperado de https://www.semanticscholar. org/paper/Nature-and-the-Life-Course\%3A-Pathways-from-Childhood-Wells-Lekies/1c94a87f065007b3e015 f83d18674006f427d951 
Whitburn, J., Linklater, W., \& Abrahamse, W. (2019). Meta-analysis of human connection to nature and proenvironmental behavior. Conservation Biology, 34(1), 180-193. https://doi.org/10.1111/cobi.13381

World, Leisure and Recreation, WLRA. (1994). International Charter for leisure education. World, Leisure and Recreation, 36(2), 41-45. https://doi.org/10.1080/10261133.1994.9673916

Zaradic, P. \& Pergams, O. (2007). Videophilia: Implications for childhood development and conservation. The Journal of Developmental Processes, 2(1), 130-144. Recuperado de https://naaee.org/eepro/research/library/videophilia-implications-childhood 